\title{
Hermite Wavelet Method for Fractional Delay Differential Equations
}

\author{
Umer Saeed and Mujeeb ur Rehman \\ School of Natural Sciences, National University of Sciences and Technology, Sector H-12, Islamabad, Pakistan \\ Correspondence should be addressed to Umer Saeed; umer.math@gmail.com
}

Received 4 April 2014; Accepted 12 June 2014; Published 2 July 2014

Academic Editor: Honglei Xu

Copyright (C) 2014 U. Saeed and M. ur Rehman. This is an open access article distributed under the Creative Commons Attribution License, which permits unrestricted use, distribution, and reproduction in any medium, provided the original work is properly cited.

We proposed a method by utilizing method of steps and Hermite wavelet method, for solving the fractional delay differential equations. This technique first converts the fractional delay differential equation to a fractional nondelay differential equation and then applies the Hermite wavelet method on the obtained fractional nondelay differential equation to find the solution. Several numerical examples are solved to show the applicability of the proposed method.

\section{Introduction}

The future state of a physical system depends not only on the present state but also on its past history. Functional differential equations provide a mathematical model for such physical systems in which the rate of change of the system may depend on the influence of its hereditary effects. Delay differential equations have numerous applications in mathematical modeling [1], for example, physiological and pharmaceutical kinetics, chemical kinetics, the navigational control of ships and aircrafts, population dynamics, and infectious diseases.

Delay differential equation is a generalization of the ordinary differential equation, which is suitable for physical system that also depends on the past data. During the last decade, several papers have been devoted to the study of the numerical solution of delay differential equations. Therefore different numerical methods [2-7] have been developed and applied for providing approximate solutions.

Method of steps is easy to understand and implement. In the method of steps [8], we convert the delay differential equation to a nondelay differential equation. The method of steps is utilized in [9] for solving integer order delay differential equations.

Hermite wavelet method [10] is implemented for finding the numerical solution of the boundary value problems and compares the obtained solutions with exact solution. In [11], authors utilized the physicists Hermite wavelet method for solving linear singular differential equations. According to our information, Hermite wavelet method has not been implemented for delay differential equations.

In the present work, we established a technique by combining both the method of steps and the Hermite wavelets method for solving the fractional delay differential equation. We also implemented the Hermite wavelet method for solving fractional delay differential equation, as described in Example 6, which was not implemented before. Shifted Chebyshev nodes are used as the collocation points. Comparison of solutions by these two methods, proposed method and Hermite wavelet method, with each other and with exact solution are also presented.

\section{Preliminaries}

2.1. Hermite Wavelets. The Hermite polynomials $H_{m}(x)$, of order $m$, are defined on the interval $[-\infty, \infty]$ and given by the following recurrence formulae:

$$
\begin{gathered}
H_{0}(x)=1, \quad H_{1}(x)=2 x, \\
H_{m+1}(x)=2 x H_{m}(x)-2 m H_{m-1}(x), \quad m=1,2,3, \ldots
\end{gathered}
$$


The polynomials $H_{m}(x)$ are orthogonal with respect to the weight function $e^{-x^{2}}$; that is,

$$
\int_{-\infty}^{\infty} e^{-x^{2}} H_{m}(x) H_{n}(x) d x= \begin{cases}0, & m \neq n \\ n ! 2^{n} \sqrt{\pi}, & m=n .\end{cases}
$$

The discrete wavelets transform is defined as

$$
\psi_{j, k}(x)=2^{j / 2} \psi\left(2^{j} x-k\right) .
$$

The set $\psi_{j, k}$ forms an orthogonal basis of $L_{2}(\mathbb{R})$. That is,

$$
\left\langle\psi_{j, k}(x), \psi_{l, m}(x)\right\rangle=\delta_{j l} \delta_{k m} .
$$

The Hermite wavelets are defined on interval $[0,1)$ by

$$
\begin{aligned}
& \psi_{n, m}(x) \\
& = \begin{cases}2^{k / 2} \sqrt{\frac{1}{n ! 2^{n} \sqrt{\pi}}} H_{m}\left(2^{k} x-\widehat{n}\right), & \frac{\widehat{n}-1}{2^{k}} \leq x<\frac{\widehat{n}+1}{2^{k}}, \\
0, & \text { elsewhere, }\end{cases}
\end{aligned}
$$

where $k=1,2,3, \ldots$, is the level of resolution, $n=$ $1,2,3, \ldots, 2^{k-1}, \widehat{n}=2 n-1$, is the translation parameter, $m=1,2, \ldots, M-1$ is the order of the Hermite polynomials, $M>0$.

2.2. Function Approximations. We can expand any function $y(x) \in L^{2}[0,1)$ into truncated Hermite wavelet series as

$$
y(x) \approx \sum_{n=1}^{2^{k-1}} \sum_{m=0}^{M-1} a_{n m} \psi_{n, m}(x)=\mathbf{a}^{T} \Psi(x)
$$

where a and $\Psi$ are $\widehat{m} \times 1\left(\widehat{m}=2^{k-1} M\right)$ matrices, given by

$$
\begin{gathered}
\mathbf{a}=\left[a_{10}, a_{11}, \ldots, a_{1 M-1}, a_{20}, a_{21}, \ldots, a_{2 M-1}, \ldots,\right. \\
\left.a_{2^{k-1} 0}, a_{2^{k-1} 1}, \ldots, a_{2^{k-1} M-1}\right]^{T}, \\
\Psi(x)=\left[\psi_{1,0}(x), \psi_{1,1}(x), \ldots, \psi_{1 M-1}(x), \psi_{2,0}(x),\right. \\
\psi_{2,1}(x), \ldots, \psi_{2, M-1}(x), \ldots, \\
\left.\psi_{2^{k-1}, 0}(x), \psi_{2^{k-1}, 1}(x), \ldots, \psi_{2^{k-1}, M-1}(x)\right]^{T} .
\end{gathered}
$$

\section{Convergence Analysis}

Let $L^{2}([0,1])$ be a Hilbert space for which $\psi_{n, m}(x)$ form an orthonormal sequence in $L^{2}([0,1])$. Let $y(x) \in L^{2}([0,1])$; we have

$$
y(x) \approx \sum_{n=1}^{2^{k-1}} \sum_{m=0}^{M-1} a_{n m} \psi_{n, m}(x),
$$

where $a_{n m}=\left\langle y(x), \psi_{n, m}(x)\right\rangle$ is an inner product of $y(x)$ and $\psi_{n, m}(x)$. Equation (8) can be written as

$$
y(x) \approx \sum_{n=1}^{2^{k-1}} \sum_{m=0}^{M-1}\left\langle y(x), \psi_{n, m}(x)\right\rangle \psi_{n, m}(x) .
$$

For simplicity, let $j=M(n-1)+m+1$; we can write (9) as

$$
y(x)=\sum_{j=1}^{\widehat{m}}\left\langle y(x), \psi_{j}(x)\right\rangle \psi_{j}(x)=\sum_{j=1}^{\widehat{m}} a_{j} \psi_{j}(x)=\mathbf{a}^{T} \Psi(x),
$$

where $a_{j}=a_{m n}, \psi_{j}(x)=\psi_{m, n}(x), \widehat{m}=2^{k-1} M$, and $\mathbf{a}=$ $\left[a_{1}, a_{2}, \ldots, a_{\widehat{m}}\right]^{T} ; \Psi(x)=\left[\psi_{1}(x), \psi_{2}(x), \ldots, \psi_{\widehat{m}}(x)\right]^{T}$.

By following the procedure in [12], we obtained the convergence of all the orthogonal wavelet methods for all levels of resolution $k$; that is, $\sum_{j=1}^{\widehat{m}} a_{j} \psi_{j}(x)$ converges to $y(x)$, as $\widehat{m} \rightarrow \infty$.

Since $\widehat{m}=2^{k-1} M$ and method converges if $\widehat{m} \rightarrow \infty$; that is, when we use higher order Hermite polynomials $M-1$ or use higher level of resolution $k$ or use both higher $M$ and $k$, we get more accurate results.

\section{Procedure for Implementation of Proposed Scheme}

The method of steps [8] is used to convert the discrete delay differential equations to nondelay differential equations on a given interval. Consider the following fractional delay differential equation with discrete delay:

$$
\begin{gathered}
{ }^{c} D^{\alpha} y(x) \\
=g(x)+f\left(y(x), y^{\prime}(x), y(q x-\tau), y^{\prime}(q x-\tau)\right), \\
a \leq x \leq b, \quad 1<\alpha \leq 2, \\
y(x)=\phi(x), \quad-b \leq x \leq a,
\end{gathered}
$$

where $g(x)$ is a source function and $f$ is a continuous linear or nonlinear function. Also $q$ is constant, $\tau$ is delay, and $q x-\tau$ is called delay argument. The delay $\tau(x, y(x))$ is called constant delay, time dependent delay, and state dependent delay if the delay $\tau(x, y(x))$ is constant, function of time $x$, and function of time $x$ and $y(x)$, respectively.

Proposed method consists of two methods, method of steps and Hermite wavelet method. We first implement the method of steps to the fractional delay differential equation (11) and get the fractional nondelay differential equation by utilizing initial function, $\phi(x)$, and then we utilize the Hermite wavelet method for solving the obtained fractional nondelay differential equation.

4.1. Method of Steps. In the fractional delay differential equation the solution $y(x)$ is known on $[-b, a]$, say $\phi(x)$, and call this solution $y_{0}(x)$; that is, $y_{0}(q x-\tau)=\phi(q x-\tau)$, which is known. Now the fractional delay differential equation on $[a, b]$ takes the form

$$
\begin{array}{r}
{ }^{c} D^{\alpha} y(x) \\
=g(x)+f\left(y(x), y^{\prime}(x), y_{0}(q x-\tau), y_{0}^{\prime}(q x-\tau)\right), \\
a \leq x \leq b, \quad 1<\alpha \leq 2,
\end{array}
$$

subject to the initial conditions $y(a)=\phi(a), y^{\prime}(a)=\phi^{\prime}(a)$. 
It is a fractional nondelay differential equation because $y_{0}(q x-\tau)$ and $y_{0}^{\prime}(q x-\tau)$ are known.

4.2. Hermite Wavelet Method for Fractional Nondelay Differential Equation. We solve the obtained fractional nondelay differential equation (12) on $[a, b]$ by using the Hermite wavelet method. The procedure for implementation of Hermite wavelet method for fractional differential equation is as follows.

Step 1. Approximate the unknown function $y(x)$ of (12) by the Hermite wavelet method as

$$
y(x) \simeq \sum_{n=1}^{2^{k-1}} \sum_{m=0}^{M-1} a_{n m} \psi_{n, m}(x) .
$$

Step 2. Substitute (13) in (12) to get the residual

$$
\begin{gathered}
\sum_{n=1}^{2^{k-1}} \sum_{m=0}^{M-1} a_{n m}^{c} D^{\alpha} \psi_{n, m}(x) \\
=g(x)+f\left(\sum_{n=1}^{2^{k-1}} \sum_{m=0}^{M-1} a_{n m} \psi_{n, m}(x), \sum_{n=1}^{2^{k-1} M-1} \sum_{m=0} a_{n m} \psi_{n, m}^{\prime}(x),\right. \\
\left.y_{0}(q x-\tau), y_{0}^{\prime}(q x-\tau)\right) .
\end{gathered}
$$

Step 3. Set the residual (14) to be equal to zero at the set of Chebyshev nodes, $x_{i}=((b-a) / 2) \cos \left((2 i+1) \pi / 2^{k} M\right)+(a+$ $b) / 2, i=0,1,2, \ldots, M-1$, on interval $[a, b]$. Consider

$$
\begin{gathered}
\sum_{n=1}^{2^{k-1}} \sum_{m=0}^{M-1} a_{n m}^{c} D^{\alpha} \psi_{n, m}\left(x_{i}\right)-g\left(x_{i}\right) \\
-f\left(\sum_{n=1}^{2^{k-1}} \sum_{m=0}^{M-1} a_{n m} \psi_{n, m}\left(x_{i}\right), \sum_{n=1}^{2^{k-1}} \sum_{m=0}^{M-1} a_{n m} \psi_{n, m}^{\prime}\left(x_{i}\right),\right. \\
\left.y_{0}\left(q x_{i}-\tau\right), y_{0}^{\prime}\left(q x_{i}-\tau\right)\right)=0 .
\end{gathered}
$$

We obtain $2^{k-1} M-p$ equations, where $p$ is the number of conditions of the delay equation. According to (11), two conditions are given, $p=2$, so we get $2^{k-1} M-2$ equations from (15) by using Chebyshev nodes $x_{i}$. Two more equations are obtained from the conditions of (11); that is,

$$
\begin{aligned}
& y(a)=\phi(a), \\
& \Longrightarrow \sum_{n=1}^{2^{k-1}} \sum_{m=0}^{M-1} a_{n m} \psi_{n, m}(a)=\phi(a), \\
& y^{\prime}(a)=\phi^{\prime}(a), \\
& \Longrightarrow \sum_{n=1}^{2^{k-1}} \sum_{m=0}^{M-1} a_{n m} \psi_{n, m}^{\prime}(a)=\phi^{\prime}(a) .
\end{aligned}
$$

We obtained $2^{k-1} M$ equations either linear or nonlinear along with $2^{k-1} M$ unknown coefficients $a_{n m}$, which is solved by Newton iterative method to get $a_{n m}$ 's and use it in (13) to get the approximate solution. Denote the obtained solution as $y_{1}(x)$, which is defined on $[a, b]$.

Continue the procedure for the subsequent interval; delay differential equation on $[b, 2 b]$ becomes

$$
\begin{aligned}
&{ }^{c} D^{\alpha} y(x) \\
&=h(x)+f\left(y(x), y^{\prime}(x),\right.\left.y_{1}(q x-\tau), y_{1}^{\prime}(q x-\tau)\right), \\
& b \leq x \leq 2 b, \quad 1<\alpha \leq 2,
\end{aligned}
$$

subject to the initial conditions $y(b)=y_{1}(b), y^{\prime}(b)=y_{1}^{\prime}(b)$, which is again a fractional nondelay differential equation and solve it by the Hermite wavelet method to get $y_{2}(x)$ on $[b, 2 b]$. This procedure may be continued for subsequent intervals.

\section{Numerical Solutions}

In this section, we utilize the proposed scheme for finding the numerical solution of linear and nonlinear fractional delay differential equations. The notations $y_{\text {pro }}, y_{\text {exact }}$, and $E_{\text {abs }}$ represent the solution by proposed method, exact solution, and their absolute error, respectively. We use the results up to 100 decimal places. Through this work we use Caputo derivatives. For the details of fractional derivatives and integrals we refer the readers to [13].

\subsection{Linear Delayed Fractional Differential Equations}

Example 1. Consider the fractional delay differential equation

$$
\begin{gathered}
{ }^{c} D^{\alpha} y(x)+y(x)-y(x-\tau) \\
=\frac{2}{\Gamma(3-\alpha)} x^{2-\alpha}-\frac{1}{\Gamma(2-\alpha)} x^{1-\alpha}+2 \tau x-\tau^{2}-\tau, \\
\quad x>0, \quad 0<\alpha<1, \\
y(x)=0, \quad x \leq 0 .
\end{gathered}
$$

The exact solution [2], when $\alpha=1$, is $y(x)=x^{2}-x$.

The results obtained by the proposed method, by taking $\alpha=0.5, k=1, M=3$, and $\tau=0.01 e^{-x}$, are shown in Figure 1 along with the exact solution. Table 1 indicates that results obtained from the proposed method are closer to exact solution and better than the method [2]. $E_{\mathrm{abs}}$ and error represent the absolute error by proposed method and method [2], respectively.

Example 2. Consider the following fractional delay differential equation:

$$
\begin{array}{r}
{ }^{c} D^{\alpha} y(x)=-y(x)-y(x-0.3)+e^{-x+0.3}, \\
0 \leq x \leq 1, \quad 2<\alpha \leq 3,
\end{array}
$$

subject to the initial conditions $y(0)=1, y^{\prime}(0)=-1$, and $y^{\prime \prime}(0)=1$. The exact solution, when $\alpha=1$, is $y(x)=e^{-x}$. 
TABLE 1: Comparison of the solution by proposed method at $M=3$, with Exact solution and method [2].

\begin{tabular}{lcccccr}
\hline$x$ & \multicolumn{2}{c}{$\tau=0.1, \alpha=0.2$} & \multicolumn{2}{c}{$\tau=0.01 e^{-x}, \alpha=0.9$} & \multicolumn{2}{c}{$\tau=0.01 e^{-x}, \alpha=0.2$} \\
& $E_{\mathrm{abs}}$ & Error [2] & $E_{\mathrm{abs}}$ & Error [2] & $E_{\mathrm{abs}}$ & Error [2] \\
\hline 5 & $1.9000 E-97$ & 0.0062 & $4.0000 E-98$ & 0.0010 & $2.7000 E-97$ & 0.0074 \\
10 & $2.7000 E-97$ & 0.0134 & $1.1000 E-97$ & $4.7115 E-4$ & $4.0000 E-97$ & 0.0082 \\
50 & $3.0000 E-96$ & 0.0690 & $0.0000 E+00$ & $7.0303 E-4$ & $5.0000 E-96$ & 0.0052 \\
\hline
\end{tabular}

TABLE 2: Comparison of the solution by proposed method, $M=25$, with Exact solution and Adomian decomposition method [3], at $\alpha=3$.

\begin{tabular}{lcccc}
\hline$x$ & $y_{\text {pro }}$ & $y_{\text {exact }}$ & $E_{\text {abs }}$ & $E_{\text {ADM }}[3]$ \\
\hline 0.0 & 1.0000 & 1.0000 & $0.00 E+00$ & $8.52 E-14$ \\
0.2 & 0.8187 & 0.8187 & $9.12 E-42$ & $3.83 E-14$ \\
0.4 & 0.6703 & 0.6703 & $6.90 E-41$ & $1.68 E-13$ \\
0.6 & 0.5488 & 0.5488 & $2.38 E-40$ & $6.00 E-14$ \\
0.8 & 0.4493 & 0.4493 & $6.01 E-41$ & $6.66 E-15$ \\
1.0 & 0.3679 & 0.3679 & $3.58 E-36$ & $4.57 E-14$ \\
\hline
\end{tabular}

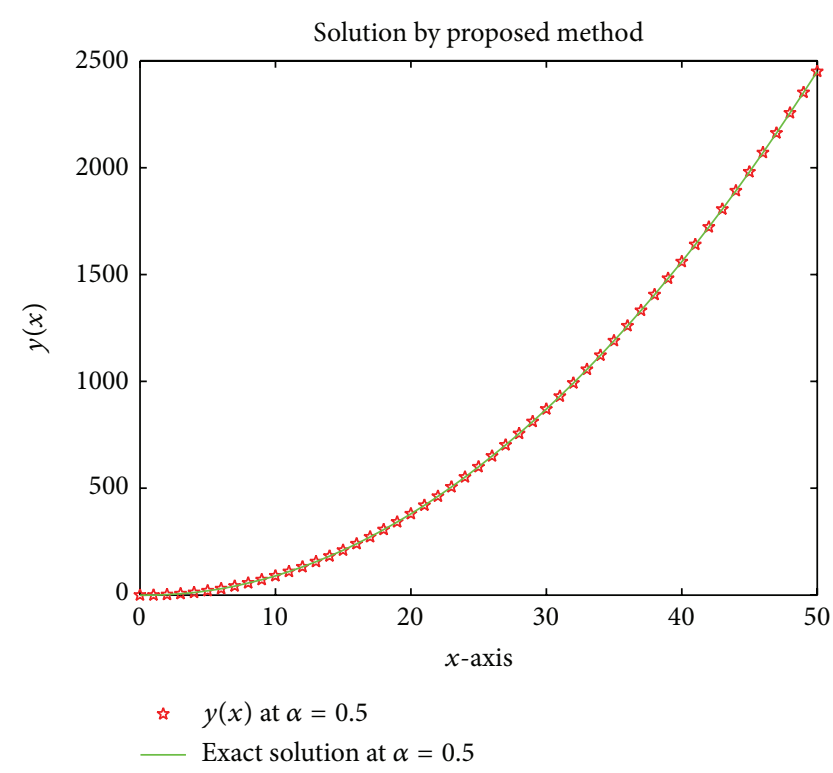

FIGURE 1: Solutions by the proposed method, $\tau=0.01 e^{-x}$, and exact solutions, at $\alpha=0.5$.

According to Table 2, proposed method provides more accurate results as compared to Adomian decomposition method [3]. These results are obtained by fixing $M=25$ and $k=1$, at $\alpha=3$. Solutions by the proposed method at different values of $\alpha$ are shown in Figure 2, which shows that solutions by proposed method at different $\alpha$ converge to the exact solution at $\alpha=3$, when $\alpha$ approaches to 3 .

Example 3. Consider the fractional pantograph equation

$$
\begin{gathered}
{ }^{c} D^{\alpha} y(x)=\frac{1}{2} e^{x / 2} y\left(\frac{x}{2}\right)+\frac{1}{2} y(x), \quad 0<\alpha \leq 1,0 \leq x \leq 1, \\
y(0)=1 .
\end{gathered}
$$

The exact solution, when $\alpha=1$, is $y(x)=e^{x}$.

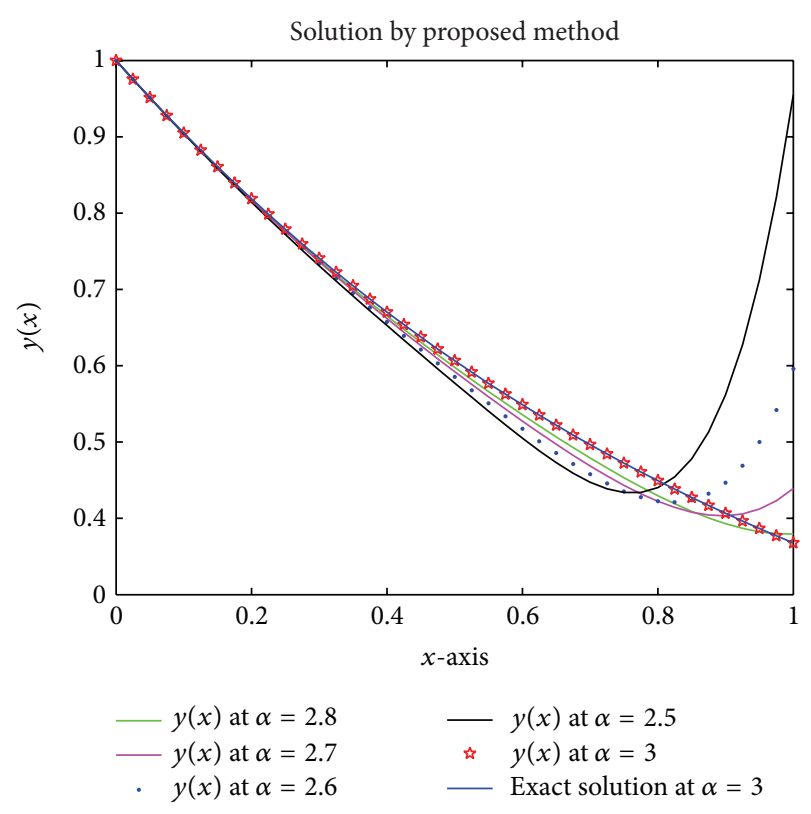

FIgURE 2: Solutions by the proposed method at different $\alpha, M=7$, $k=1$, and exact solution at $\alpha=3$.

By fixing $k=1$ and $M=4$, we plot the solutions by proposed method at different values of $\alpha$ and exact solution at $\alpha=1$, as shown in Figure 3. It shows that proposed solution approaches to the exact solution while $\alpha$ approaches to 1 .

Comparison of proposed solution $y_{\text {pro }}$ at $\alpha=1, M=30$, and $k=1$, with Adomian decomposition method [3] and the spline function technique [4], is shown in Table 3. The notations $E_{\mathrm{abs}}, E_{\mathrm{ADM}}$, and $E_{\text {spline }}$ represent the absolute error by proposed method, Adomian decomposition method, and the spline function technique, respectively. We can get more accurate results while increasing $M$. 
TABLE 3: Solution by proposed method for $M=30, k=1$.

\begin{tabular}{lccccr}
\hline$x$ & & $\alpha=1$ & $E_{\text {ADM }}[3]$ & 0.00 \\
0 & $y_{\text {exact }}$ & 1.22140 & $1.96519 E-51$ & $2.22 E-16$ & $3.10 E-15$ \\
0.4 & 1.22140 & 1.49182 & $2.51474 E-51$ & $2.22 E-16$ & $7.54 E-15$ \\
0.6 & 1.49182 & 1.82212 & $2.56283 E-51$ & $1.33 E-15$ & $1.39 E-14$ \\
0.8 & 1.82212 & 2.22554 & $1.03409 E-50$ & $4.88 E-15$ & $2.13 E-14$ \\
1.0 & 2.22554 & 2.71828 & $8.81662 E-49$ & & $3.19 E-14$ \\
\hline
\end{tabular}

TABLE 4: Absolute errors using proposed method at $\alpha=1$ and $M=17$.

\begin{tabular}{lcccc}
\hline $\begin{array}{l}\alpha=1 \\
x\end{array}$ & SLC method [5] & RKHSM [6] & RKT method [7] & $E_{\mathrm{abs}}$ \\
\hline 0.1 & $4.27 E-17$ & $1.42 E-4$ & $8.68 E-4$ & $1.23 E-24$ \\
0.2 & $2.70 E-17$ & $1.17 E-4$ & $1.49 E-3$ & $3.41 E-25$ \\
0.3 & $5.94 E-17$ & $9.45 E-4$ & $1.90 E-3$ & $1.74 E-24$ \\
0.4 & $8.01 E-17$ & $7.59 E-4$ & $2.16 E-3$ & $2.79 E-24$ \\
0.5 & $8.27 E-17$ & $6.03 E-4$ & $2.28 E-3$ & $3.33 E-24$ \\
0.6 & $1.95 E-16$ & $4.73 E-4$ & $2.31 E-3$ & $3.94 E-24$ \\
0.7 & $1.56 E-16$ & $3.64 E-4$ & $2.27 E-3$ & $3.56 E-24$ \\
0.8 & $8.80 E-17$ & $2.75 E-4$ & $2.17 E-3$ & $2.35 E-25$ \\
0.9 & $1.03 E-16$ & $2.03 E-4$ & $2.03 E-3$ & $1.02 E-23$ \\
1.0 & $1.23 E-16$ & $1.43 E-4$ & $1.86 E-3$ & $1.58 E-22$ \\
\hline
\end{tabular}

Example 4. Consider the following fractional neutral functional differential equation with proportional delay:

$$
\begin{aligned}
& { }^{c} D^{\alpha} y(x) \\
& =-y(x)+0.1 y(0.8 x)+0.5^{c} D^{\alpha} y(0.8 x) \\
& +(0.32 x-0.5) e^{-0.8 x}+e^{-x}, \quad x \geq 0,0<\alpha \leq 1, \\
& y(0)=0,
\end{aligned}
$$

which has the exact solution, when $\alpha=1$, which is $x e^{x}$.

We implement the proposed method by fixing $k=$ $1, M=5$, at different values of $\alpha$, the results are shown in Figure 4 along with the exact solution at $\alpha=1$. Table 4 shows that proposed method gives more accurate results as compared to the spectral shifted Legendre-Gauss collocation (SLC) method [5], the reproducing kernel Hilbert space method (RKHSM) [6], and a Runge-Kutta-type (RKT) method [7].

\subsection{Nonlinear Delayed Fractional Differential Equations}

Example 5. Consider the fractional nonlinear delay differential equation

$$
{ }^{c} D^{\alpha} y(x)=1-2 y^{2}\left(\frac{x}{2}\right), \quad 0 \leq x \leq 1,1<\alpha \leq 2,
$$

subject to the initial conditions, $y(0)=1$ and $y^{\prime}(0)=0$. The exact solution [14], when $\alpha=2$, is $y(x)=\cos (x)$.

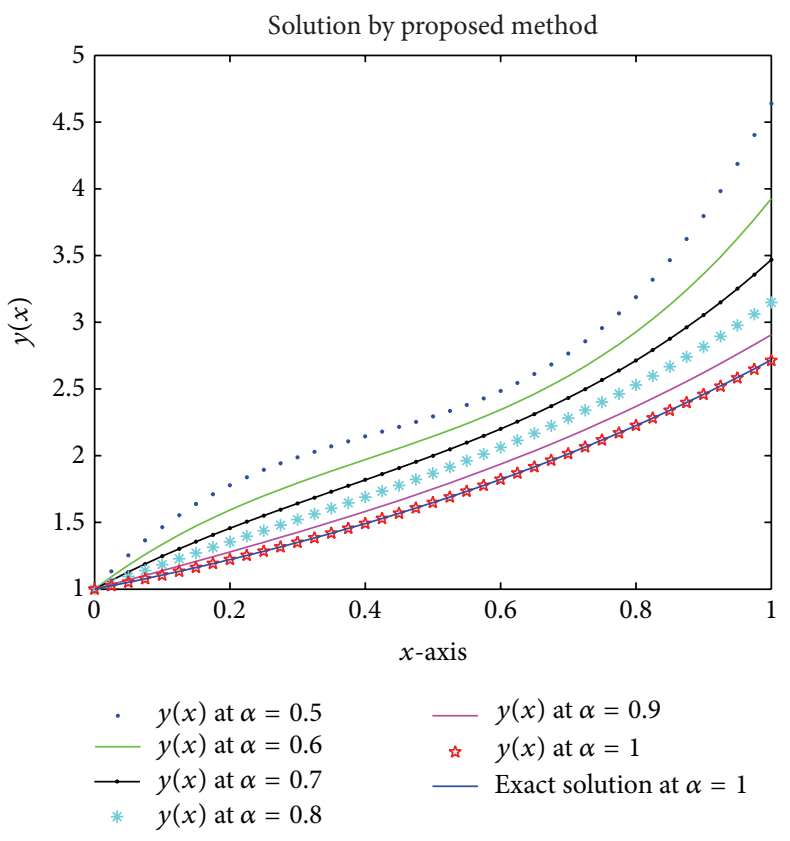

FIGURE 3: Solutions by the proposed method at different $\alpha, M=4$, $k=1$, and exact solutions at $\alpha=1$.

Solution by proposed method at different values of $\alpha$ is plotted in Figure 5, which shows that proposed solution converges to the exact solution when $\alpha$ approaches to 2 . According to Table 5, absolute error reduces while increasing $M$. 


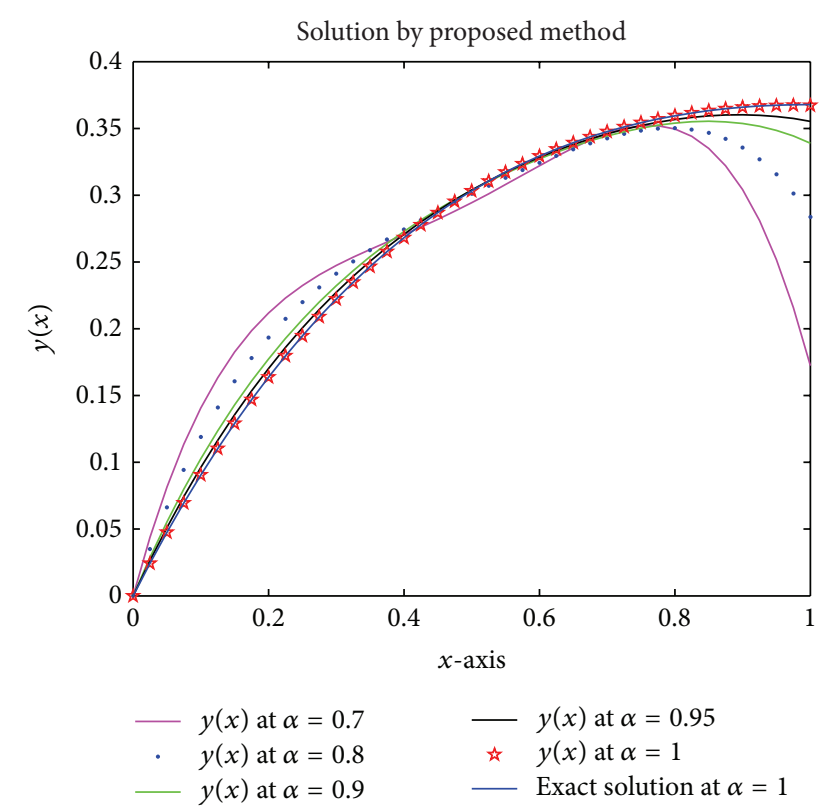

FIgURE 4: Solution by the proposed method at different $\alpha, M=5$ and exact solution at $\alpha=1$.

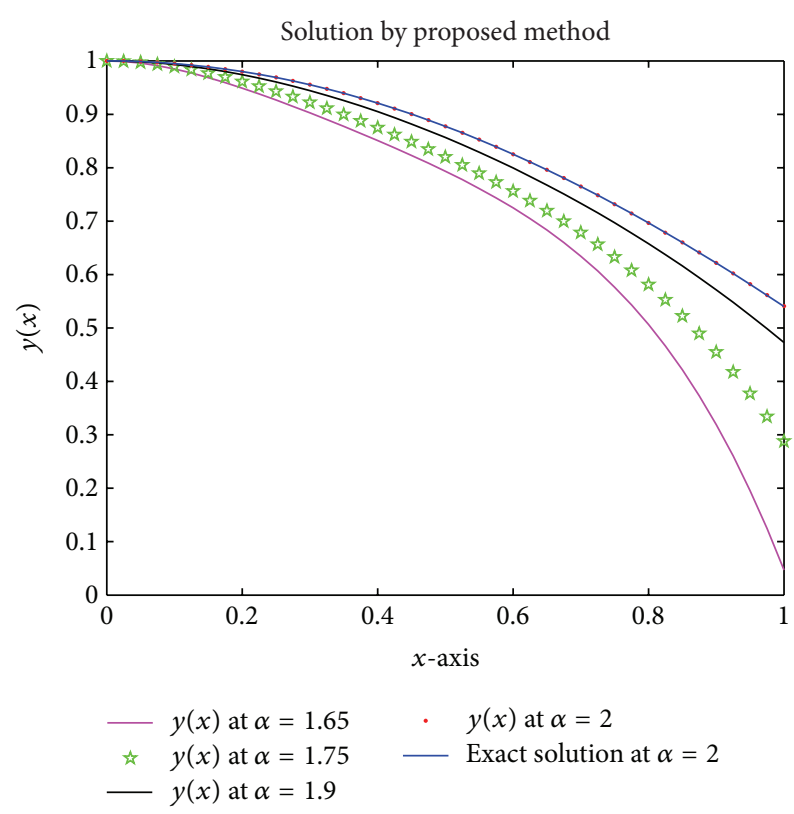

Figure 5: Solution by the proposed method at different $\alpha, M=5$ and exact solution at $\alpha=2$.

\subsection{Comparison of Proposed Method and Hermite Wavelet Method}

Example 6. Consider the fractional nonlinear neutral delay differential equation

$$
\begin{array}{r}
{ }^{c} D^{\alpha} y(x)=\frac{1}{2} y(x)+\frac{1}{2} y\left(\frac{x}{2}\right){ }^{c} D^{\alpha} y\left(\frac{x}{2}\right), \\
x \geq 0, \quad 0<\alpha \leq 1,
\end{array}
$$

subject to the initial conditions, $y(0)=1$. The exact solution [15], when $\alpha=1$, is $y(x)=e^{x}$.

\subsection{Hermite Wavelet Method for Fractional Delay Differential} Equation. We can approximate the solution of (23) by the Hermite wavelet method as

$$
y(x) \simeq \sum_{n=1}^{2^{k-1}} \sum_{m=0}^{M-1} a_{n m} \psi_{n, m}(x)
$$

In the delay equations, we also have to approximate the delay unknown function $y(x / 2)$ in terms of the Hermite wavelet series at delay time as

$$
y\left(\frac{x}{2}\right) \simeq \sum_{n=1}^{2^{k-1}} \sum_{m=0}^{M-1} a_{n m} \psi_{n, m}\left(\frac{x}{2}\right)
$$

We call this series as the delay Hermite wavelet series. Substituting (24) and (25) in (23), we get the residual as

$$
\begin{aligned}
\sum_{n=1}^{2^{k-1}} \sum_{m=0}^{M-1} a_{n m}^{c} D^{\alpha} \psi_{n, m}(x) & \\
= & \frac{1}{2} \sum_{n=1}^{2^{k-1}} \sum_{m=0}^{M-1} a_{n m} \psi_{n, m}(x)+\frac{1}{2} \sum_{n=1}^{2^{k-1}} \sum_{m=0}^{M-1} a_{n m} \psi_{n, m}\left(\frac{x}{2}\right) \\
& \times\left(\sum_{n=1}^{2^{k-1} M-1} \sum_{m=0}^{c} a_{n m}^{c} D^{\alpha} \psi_{n, m}\left(\frac{x}{2}\right)\right) .
\end{aligned}
$$

Set the residual (26) to be equal to zero at the set of Chebyshev nodes, $x_{i}=(1 / 2) \cos \left((2 i+1) \pi / 2^{k} M\right)+(1 / 2)$, $k=0,1,2, \ldots, M-1$, on interval $[0,1]$, we get

$$
\begin{aligned}
& \sum_{n=1}^{2^{k-1}} \sum_{m=0}^{M-1} a_{n m}^{c} D^{\alpha} \psi_{n, m}\left(x_{i}\right)-\frac{1}{2} \sum_{n=1}^{2^{k-1}} \sum_{m=0}^{M-1} a_{n m} \psi_{n, m}\left(x_{i}\right) \\
& \quad-\frac{1}{2} \sum_{n=1}^{2^{k-1}} \sum_{m=0}^{M-1} a_{n m} \psi_{n, m}\left(\frac{x_{i}}{2}\right) \\
& \quad \times\left(\sum_{n=1}^{2^{k-1} M-1} \sum_{m=0}^{c} a_{n m}^{c} D^{\alpha} \psi_{n, m}\left(\frac{x_{i}}{2}\right)\right)=0 .
\end{aligned}
$$

We get $2^{k-1} M-1$ equations from (27) by using Chebyshev nodes $x_{i}$. One more equation is obtained from the condition of (23); that is,

$$
\begin{aligned}
& y(0)=1 \\
& \Longrightarrow \sum_{n=1}^{2^{k-1}} \sum_{m=0}^{M-1} a_{n m} \psi_{n, m}(0)=1
\end{aligned}
$$

We obtained $2^{k-1} M$ nonlinear equations along with $2^{k-1} M$ unknown coefficients $a_{n m}$, which is solved by Newton iterative method to get $a_{n m}$ 's and used in (24) to get the approximate solution by Hermite wavelet method. 
TABLE 5: Absolute errors by using proposed method at different $M$ and $\alpha=2$.

\begin{tabular}{|c|c|c|c|c|}
\hline $\begin{array}{l}\alpha=2 \\
x\end{array}$ & $\begin{array}{c}M=15 \\
E_{\mathrm{abs}}\end{array}$ & $\begin{array}{c}M=25 \\
E_{\text {abs }}\end{array}$ & $\begin{array}{c}M=30 \\
E_{\mathrm{abs}}\end{array}$ & $\begin{array}{c}M=40 \\
E_{\mathrm{abs}}\end{array}$ \\
\hline 0.1 & $2.17077 E-23$ & $1.96989 E-42$ & $8.02964 E-52$ & $5.46682 E-60$ \\
\hline 0.2 & $4.49566 E-23$ & $2.28499 E-42$ & $7.79780 E-52$ & $1.52681 E-59$ \\
\hline 0.3 & $1.67001 E-22$ & $6.26291 E-42$ & $1.42878 E-51$ & $1.57732 E-59$ \\
\hline 0.4 & $3.60801 E-23$ & $2.25484 E-41$ & $2.38398 E-51$ & $5.49810 E-60$ \\
\hline 0.5 & $2.87200 E-22$ & $2.25484 E-41$ & $4.19542 E-51$ & $1.09577 E-58$ \\
\hline 0.6 & $4.60073 E-24$ & $7.94684 E-41$ & $6.75256 E-51$ & $9.58443 E-59$ \\
\hline 0.7 & $4.89152 E-22$ & $1.18456 E-40$ & $1.12211 E-50$ & $3.69647 E-57$ \\
\hline 0.8 & $1.23582 E-21$ & $1.36081 E-40$ & $1.58769 E-50$ & $1.69940 E-55$ \\
\hline 0.9 & $5.88496 E-21$ & $4.92247 E-40$ & $5.35881 E-50$ & $5.29603 E-54$ \\
\hline 1.0 & $5.30153 E-19$ & $1.25319 E-37$ & $1.99293 E-47$ & $3.70755 E-53$ \\
\hline
\end{tabular}

TABle 6: Comparison of proposed and Hermite wavelet methods $M=9, k=1$.

\begin{tabular}{lccccc}
\hline & & & $\alpha=1$ & \\
$x$ & $y_{\text {HWM }}$ & $y_{\text {pro }}$ & $y_{\text {exact }}$ & $E_{\text {HWM }}$ & $0.00000 E+00$ \\
0.0 & 1.0000000000 & 1.0000000000 & 1.0000000000 & $1.33288 E-11$ & $5.37737 E-12$ \\
0.1 & 1.1051709181 & 1.1051709181 & 1.1051709181 & $3.93123 E-12$ & $1.14859 E-11$ \\
0.2 & 1.2214027582 & 1.2214027581 & 1.2214027582 & $2.83949 E-11$ & $2.13830 E-11$ \\
0.3 & 1.3498588076 & 1.3498588076 & 1.3498588076 & $1.26536 E-11$ & $6.30257 E-12$ \\
0.4 & 1.4918246977 & 1.4918246976 & 1.4918246976 & $8.42958 E-12$ & $3.08447 E-11$ \\
0.5 & 1.6487212707 & 1.6487212707 & 1.6487212707 & $5.79353 E-11$ & $1.57380 E-11$ \\
0.6 & 1.8221188004 & 1.8221188004 & 1.8221188004 & $8.92374 E-11$ & $4.32148 E-11$ \\
0.7 & 2.0137527076 & 2.0137527075 & 2.0137527075 & $3.39446 E-11$ & $6.08108 E-11$ \\
0.8 & 2.2255409285 & 2.2255409284 & 2.2255409285 & $6.71791 E-11$ & $6.72473 E-11$ \\
0.9 & 2.4596031112 & 2.4596031112 & 2.4596031112 & $8.41911 E-10$ & $8.35594 E-10$ \\
1.0 & 2.7182818276 & 2.7182818276 & 2.7182818285 & & \\
\hline
\end{tabular}

We fix $M=9, k=1$, and implement the Hermite wavelet and proposed method to (23). The results are shown in Table 6 along with the absolute errors. According to Table 6, Hermite wavelet and proposed methods give good results; that is, for some points proposed method is more accurate as compared to Hermite wavelet method and vice versa.

For the problem (23), run time of proposed method and Hermite wavelet method is 1.21 and 26.21 seconds, respectively. Proposed method is more efficient than the Hermite wavelet method. $y_{\mathrm{HWM}}$ and $E_{\mathrm{HWM}}$ represent the solution by Hermite wavelet method and their absolute error, respectively.

For this purpose, we use Maple 13 in system with Core Duo CPU 2.00 GHz and RAM 2.50 GB.

\section{Conclusion}

It is shown that proposed method gives excellent results when applied to different fractional linear and nonlinear delay differential equations. The results obtained from the proposed method are more accurate and better than the results obtained from other methods, as shown in Tables 1-4. The solution of the fractional delay differential equation converges to the solution of integer delay differential equation, as shown in Figures 2-5. According to the convergence analysis, error by the proposed method reduces while increasing $M$, as shown in Table 5. Table 6 indicates that both Hermite wavelet method and proposed method give good results. Proposed method is more efficient than the Hermite wavelet method.

\section{Conflict of Interests}

The authors declare that there is no conflict of interests regarding the publication of this paper.

\section{Acknowledgment}

The authors are grateful to the anonymous reviewers for their valuable comments which led to the improvement of the paper.

\section{References}

[1] V. Lakshmikantham and S. Leela, Differential and Integral Inequalities, Academic Press, New York, NY, USA, 1969.

[2] Z. Wang, "A numerical method for delayed fractional-order differential equations," Journal of Applied Mathematics, vol. 2013, Article ID 256071, 7 pages, 2013. 
[3] D. J. Evans and K. R. Raslan, "The Adomian decomposition method for solving delay differential equation," International Journal of Computer Mathematics, vol. 82, no. 1, pp. 49-54, 2005.

[4] A. El-Safty, M. S. Salim, and M. A. El-Khatib, "Convergence of the spline function for delay dynamic system," International Journal of Computer Mathematics, vol. 80, no. 4, pp. 509-518, 2003.

[5] A. H. Bhrawy, L. M. Assas, E. Tohidi, and M. A. Alghamdi, "A Legendre-Gauss collocation method for neutral functionaldifferential equations with proportional delays," Advances in Difference Equations, vol. 2013, article 63, 2013.

[6] X. Lv and Y. Gao, "The RKHSM for solving neutral functionaldifferential equations with proportional delays," Mathematical Methods in the Applied Sciences, vol. 36, no. 6, pp. 642-649, 2013.

[7] W. Wang, Y. Zhang, and S. Li, "Stability of continuous RungeKutta-type methods for nonlinear neutral delay-differential equations," Applied Mathematical Modelling. Simulation and Computation for Engineering and Environmental Systems, vol. 33, no. 8, pp. 3319-3329, 2009.

[8] H. Smith, An Introduction to Delay Differential Equations with Applications to the Life Sciences, Springer, New York, NY, USA, 2011.

[9] C. E. Falbo, "Some Elementary Methods for Solving Functional Differential Equations," http://www.mathfile.net/hicstat_FDE.pdf.

[10] A. Ali, M. A. Iqbal, and S. T. Mohyud-Din, "Hermite wavelets method for boundary value problems," International Journal of Modern Applied Physics, vol. 3, no. 1, pp. 38-47, 2013.

[11] M. Usman and S. T. Mohyud-Din, "Physicists Hermite wavelet method for singular differential equations," International Journal of Advances in Applied Mathematics and Mechanics, vol. 1, no. 2, pp. 16-29, 2013.

[12] S. G. Venkatesh, S. K. Ayyaswamy, and S. Raja Balachandar, "The Legendre wavelet method for solving initial value problems of Bratu-type," Computers \& Mathematics with Applications, vol. 63, no. 8, pp. 1287-1295, 2012.

[13] I. Podlubny, Fractional Differential Equations, Academic Press, San Diego, Calif, USA, 1999.

[14] F. Karakoç and H. Bereketoğlu, "Solutions of delay differential equations by using differential transform method," International Journal of Computer Mathematics, vol. 86, no. 5, pp. 914923, 2009.

[15] M. M. Khader and S. T. Mohamed, "Numerical treatment for first order neutral delay differential equations using spline functions," Engineering Mathematics Letters, vol. 1, no. 1, pp. 3243, 2012. 


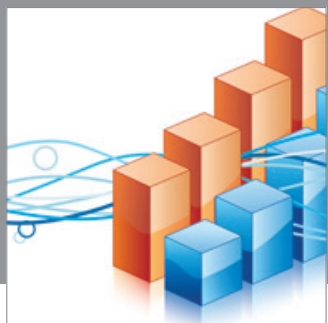

Advances in

Operations Research

mansans

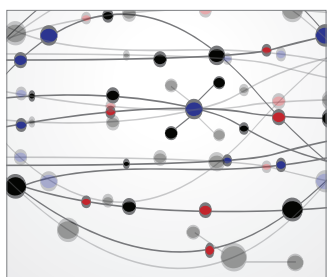

The Scientific World Journal
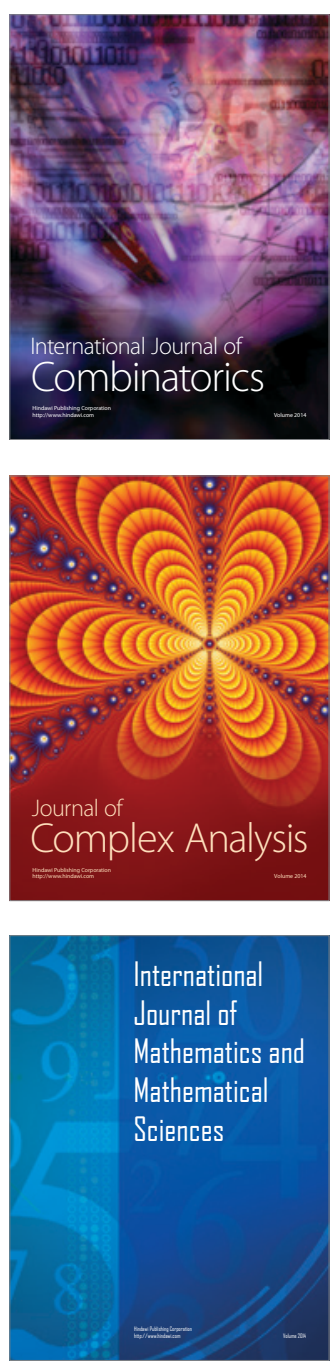
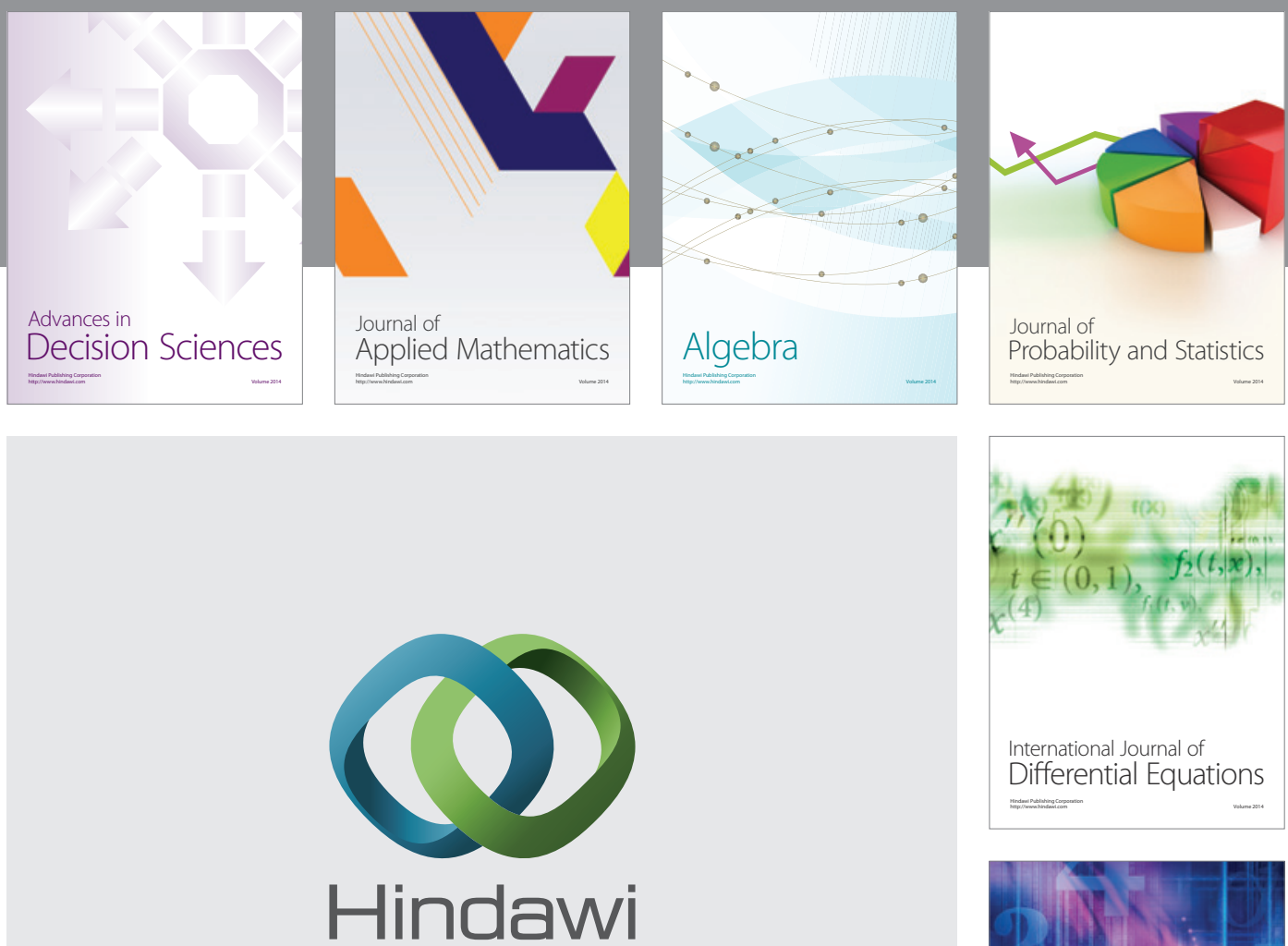

Submit your manuscripts at http://www.hindawi.com
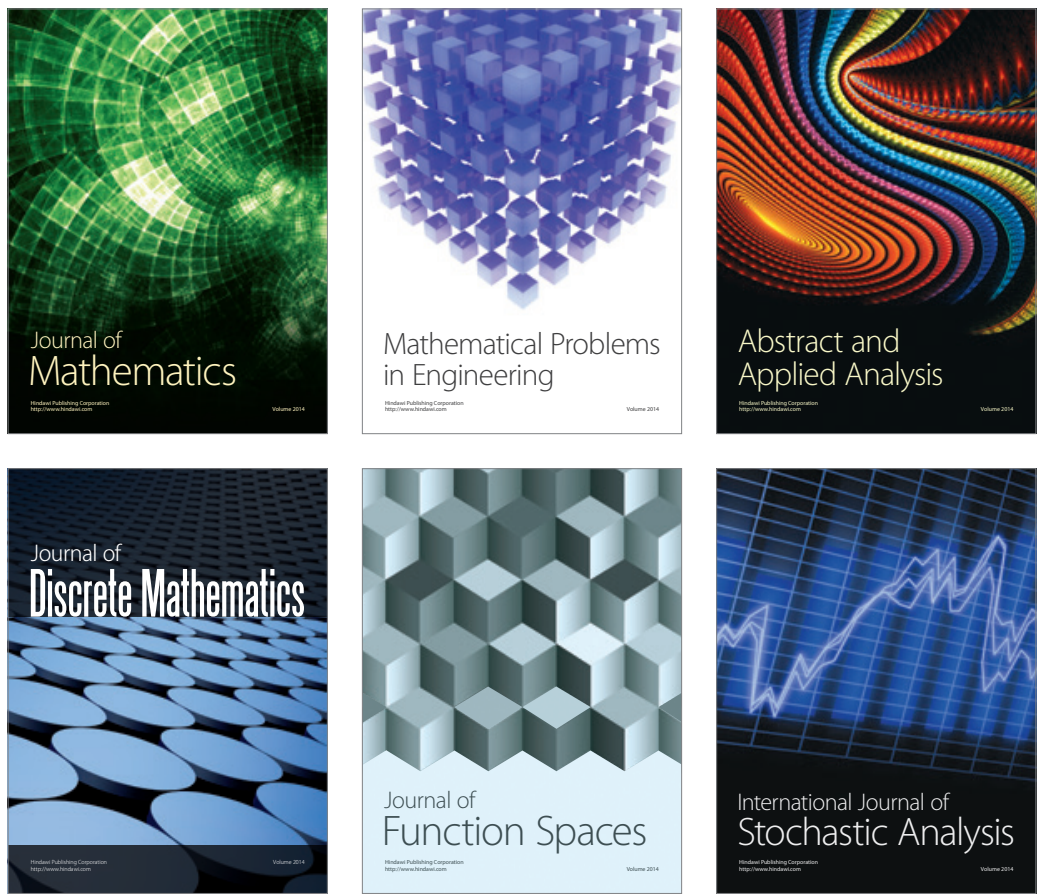

Journal of

Function Spaces

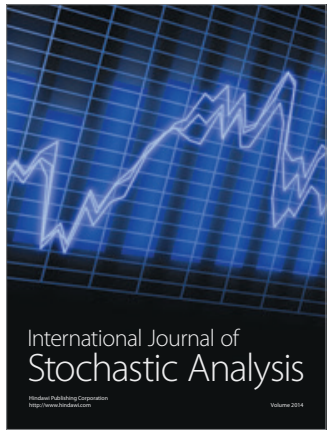

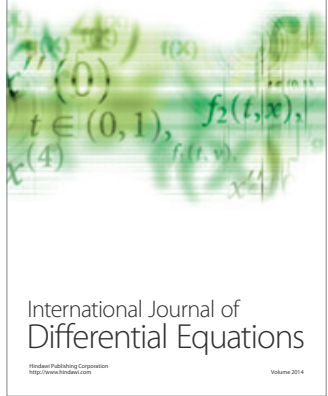
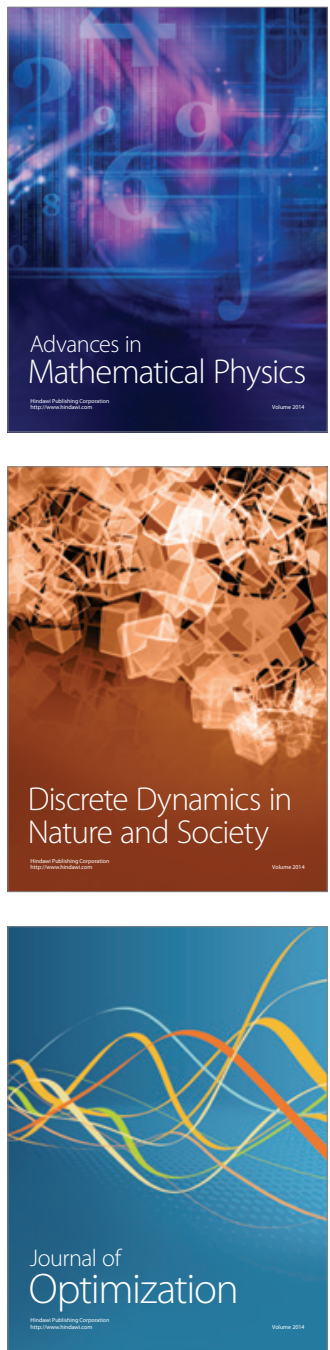